\title{
HIGHER DIMENSIONAL COHOMOLOGY OF WEIGHTED SEQUENCE ALGEBRAS
}

\author{
A. POURABBAS \\ (Received 8 October 2001; revised 10 May 2002) \\ Communicated by G. Willis
}

\begin{abstract}
It is well known that $c_{0}(\mathbb{Z})$ is amenable and so its global dimension is zero. In this paper we will investigate the cyclic and Hochschild cohomology of Banach algebra $c_{0}\left(\mathbb{Z}, \omega^{-1}\right)$ and its unitisation with coefficients in its dual space, where $\omega$ is a weight on $\mathbb{Z}$ which satisfies inf $\{\omega(i)\}=0$. Moreover we show that the weak homological bi-dimension of $c_{0}\left(\mathbb{Z}, \omega^{-1}\right)$ is infinity.
\end{abstract}

2000 Mathematics subject classification: primary 46M20, 43A15.

\section{Introduction}

The Banach algebra $\mathscr{A}$ is amenable if $\mathscr{H}^{\prime}\left(\mathscr{A}, \mathscr{X}^{\prime}\right)=0$ for every Banach $\mathscr{A}$ bimodule $\mathscr{X}$. This definition was introduced by Johnson in (1972) [8]. The Banach algebra $\mathscr{A}$ is weakly amenable if $\mathscr{H}^{1}\left(\mathscr{A}, \mathscr{A}^{\prime}\right)=0$. This definition generalizes the one which was introduced by Bade, Curtis and Dales in [1], where it was noted that a commutative Banach algebra $\mathscr{A}$ is weakly amenable if and only if $\mathscr{H}^{1}(\mathscr{A}, \mathscr{X})=0$ for every symmetric Banach $\mathscr{A}$-bimodule $\mathscr{X}$.

Johnson in [8] proved that for an amenable Banach algebra $\mathscr{A}$, the cohomology groups $\mathscr{H}^{n}\left(\mathscr{A}, \mathscr{X}^{\prime}\right)$ vanish for every Banach $\mathscr{A}$-bimodule $\mathscr{X}$ and all $n \geq 1$. The question was raised whether in general $\mathscr{H}^{n}\left(\mathscr{A}, \mathscr{A}^{\prime}\right)=0$ for a weakly amenable Banach algebra $\mathscr{A}$ and all $n \geq 1$. The question was answered in the negative in [14] by showing that $\mathscr{H}^{2}\left(\ell^{1}\left(\mathbb{F}_{2}\right), \ell^{\infty}\left(\mathbb{F}_{2}\right)\right) \neq 0$. In fact Johnson [8] showed that $\mathscr{H}^{2}\left(\ell^{1}\left(\mathbb{F}_{2}\right), \mathbb{C}\right) \neq 0$ and in [14] Sinclair and Smith showed that the non-trivial cohomology group $\mathscr{H}^{2}\left(\ell^{1}\left(\mathbb{F}_{2}\right), \mathbb{C}\right)$ is naturally embedded as a direct summand of

(C) 2003 Australian Mathematical Society $1446-7887 / 03 \$ A 2.00+0.00$ 
$\mathscr{H}^{2}\left(\ell^{1}(\mathbb{F}), \ell^{\infty}(\mathbb{F})\right)$. In this paper we will give an example of a weakly amenable Banach algebra, such that the $n^{\text {th }}$ cohomology groups with coefficients in the dual space do not vanish for all $n>1$.

It is a question of general interest whether or not the $n^{\text {th }}$ cohomology group is necessarily zero. This, and closely related questions have stimulated much of the recent development of the theory of cohomology groups.

Bade, Curtis and Dales in [1] showed that $\mathscr{H}^{1}\left(\ell^{1}\left(\mathbb{Z}_{+}\right), \ell^{1}\left(\mathbb{Z}_{+}\right)^{\prime}\right) \neq 0$. This may lead one to believe that $\mathscr{H}^{n}\left(\ell^{1}\left(\mathbb{Z}_{+}\right), \ell^{1}\left(\mathbb{Z}_{+}\right)^{\prime}\right)$ for all $n \geq 2$ are also non-zero. However, Johnson showed in [10] that the alternating cohomology of $\ell^{1}\left(\mathbb{Z}_{+}\right)$vanishes in all dimensions strictly greater than one. Then Dales and Duncan [2, Theorem 3.2] showed that $\mathscr{H}^{2}\left(\ell^{1}\left(\mathbb{Z}_{+}\right), \ell^{1}\left(\mathbb{Z}_{+}\right)^{\prime}\right)=0$. Gourdeau and White in [4] with a complicated proof showed that $\mathscr{H}^{3}\left(\ell^{1}\left(\mathbb{Z}_{+}\right), \ell^{1}\left(\mathbb{Z}_{+}\right)^{\prime}\right)=0$. This leads to the conjecture that all the cohomology groups of $\ell^{1}\left(\mathbb{Z}_{+}\right)$with coefficients in $\ell^{1}\left(\mathbb{Z}_{+}\right)^{\prime}$ vanish for $n>3$.

In this paper for the weakly amenable Banach algebra $\mathscr{A}^{\#}$, the unitisation of $\mathscr{A}=c_{0}\left(\mathbb{Z}, \omega^{-1}\right)$, we show that the cyclic cohomology group $\mathscr{H} \mathscr{C}^{n}\left(\mathscr{A}^{\#}\right)$ and the Hochschild cohomology group $\mathscr{H}^{n}\left(\mathscr{A}^{\#},\left(\mathscr{A}^{\#}\right)^{\prime}\right)$ are non-trivial for every $n \geq 2$.

Let $\omega$ be a weight sequence on $\mathbb{Z}$, that is, $\omega$ is a non-zero, positive valued function on $\mathbb{Z}$ such that $\omega(n) \leq 1$ for every $n \in \mathbb{Z}$. Set

$$
c_{0}\left(\mathbb{Z}, \omega^{-1}\right)=\left\{a=\left\{a_{n}\right\}: n \in \mathbb{Z}, \lim _{|n| \rightarrow \infty} \frac{\left|a_{n}\right|}{\omega(n)}=0\right\},
$$

where $c_{0}\left(\mathbb{Z}, \omega^{-1}\right)$ is a closed subalgebra of

$$
\ell^{\infty}\left(\mathbb{Z}, \omega^{-1}\right)=\left\{a=\left\{a_{n}\right\}: n \in \mathbb{Z},\|a\|_{\omega^{-1}}=\sup \left\{\frac{\left|a_{n}\right|}{\omega(n)}: n \in \mathbb{Z}\right\}<\infty\right\}
$$

and $c_{0}\left(\mathbb{Z}, \omega^{-1}\right)^{\prime}$ (the dual space of $\left.c_{0}\left(\mathbb{Z}, \omega^{-1}\right)\right)$ is equal to

$$
\ell^{1}(\mathbb{Z}, \omega)=\left\{a=\left\{a_{n}\right\}: n \in \mathbb{Z}, \sum_{n=-\infty}^{\infty}\left|a_{n}\right| \omega(n)<\infty\right\} .
$$

The element $e_{i}=\left\{\delta_{i j}\right\}_{j \in \mathbb{Z}}, i \in \mathbb{Z}$ is an idempotent, where $\delta_{i j}$ denotes the Kronecker delta. We denote the linear span of such elements by $E$, which is a dense subset of $c_{0}\left(\mathbb{Z}, \omega^{-1}\right) ;$ since if $a \in c_{0}\left(\mathbb{Z}, \omega^{-1}\right)$, then we define

$$
a^{n}=\sum_{i=-n}^{n} a_{i} e_{i}=\left\{\ldots, 0, a_{-n}, \ldots, a_{n}, 0, \ldots\right\}
$$

and

$$
\left\|a-a^{n}\right\|_{\omega^{-1}}=\sup _{|i|>|n|} \frac{\left|a_{i}\right|}{\omega(i)} \rightarrow 0 \quad \text { as }|n| \rightarrow \infty
$$


Since a commutative Banach algebra which is the closed linear span of its idempotents is weakly amenable [9], then $c_{0}\left(\mathbb{Z}, \omega^{-1}\right)$ is weakly amenable, and by [3, Proposition 1.4] $\mathscr{A}^{\#}$, the unitisation of $\mathscr{A}=c_{0}\left(\mathbb{Z}, \omega^{-1}\right)$ is also weakly amenable.

NOTE. In this paper every weight $\omega$ on $\mathbb{Z}$ which we consider must satisfy the condition $\inf \{\omega(i)\}=0$, because if $\inf \{\omega(i)\} \neq 0$, then $\omega^{-1}$ is a bounded weight and so $c_{0}\left(\mathbb{Z}, \omega^{-1}\right) \cong c_{0}(\mathbb{Z})$ which is amenable.

Throughout $\mathscr{A}^{\#}$ means the unitisation of $\mathscr{A}=c_{0}\left(\mathbb{Z}, \omega^{-1}\right)$. Let 1 be the unit element of $\mathscr{A}^{*}$. Suppose $E_{N}$ is the closed linear span of $\left\{e_{i}\right\}_{i=1}^{N}$. Then $E_{N}$ is a closed subalgebra of $\mathscr{A}^{\#}$. If $a \in \mathscr{A}^{\#}$, then $a=a^{\prime}+\alpha \mathbf{1}$, where $a^{\prime}=\left\{a_{n}^{\prime}\right\}_{n \in \mathbb{Z}}$ is in $c_{0}\left(\mathbb{Z}, \omega^{-1}\right)$ and $\alpha \in \mathbb{C}$. The norm on $\mathscr{A}^{\#}$ is defined by $\|a\|_{\omega^{-1}}=\left\|a^{\prime}\right\|_{\omega^{-1}}+|\alpha|$. Also for every $a=a^{\prime}+\alpha \mathbf{1}$ and $b=b^{\prime}+\beta 1$ in $\mathscr{A}^{\#}$ we define $a b=a^{\prime} b^{\prime}+\alpha b^{\prime}+\beta a^{\prime}+\alpha \beta 1$. Clearly $E_{N} \cong \mathbb{C}^{N}$ and since a direct sum of amenable algebras is amenable, then $E_{N}$ is an amenable closed subalgebra of $\mathscr{A}^{\#}$.

Note that for every $\phi \in \mathscr{Z}^{n}\left(\mathscr{A}^{\#},\left(\mathscr{A}^{\#}\right)^{\prime}\right)$, the space of all bounded $n$-cocycles, by [11] there exists $\psi_{N}$ in $\mathscr{C}^{n-1}\left(\mathscr{A}^{\#},\left(\mathscr{A}^{\#}\right)^{\prime}\right)$ such that

$$
\left(\phi-\delta \psi_{N}\right)\left(a_{1}, \ldots, a_{n}\right)=0 \text { if any one of } a_{1}, \ldots, a_{n} \text { lies in } E_{N} .
$$

But we will show that this is not true for the whole of $\mathscr{A}^{\#}$, in fact for every $n \geq 2$ we will find a (cyclic) cocycle $\phi \in \mathscr{Z}^{n}\left(\mathscr{A}^{\#},\left(\mathscr{A}^{\#}\right)^{\prime}\right)$ which does not co-bound.

The weak homological bi-dimension of a Banach algebra $\mathscr{A}$, denoted by wdb $\mathscr{A}$, is the smallest integer $n$ such that $\mathscr{H}^{m}\left(\mathscr{A}, X^{\prime}\right)=0$ for all Banach $\mathscr{A}$-bimodules $X$ and all $m>n$, or wdb $\mathscr{A}=\infty$ if there is no such $n$. If $\mathscr{A}$ is an amenable Banach algebra, then wdb $\mathscr{A}=0$ [7, Section 2.5]. The weak homological bi-dimension of a Banach algebra is a number that measures how much this algebra is homologically worse than amenable. The homological bi-dimension of a Banach algebra $\mathscr{A}$, denoted by $\mathrm{db} \mathscr{A}$, is the smallest integer $n$ such that $\mathscr{H}^{m}(\mathscr{A}, X)=0$ for all Banach $\mathscr{A}$-bimodules $X$ and all $m>n$, or wdb $\mathscr{A}=\infty$ if there is no such $n$. For every Banach algebra $\mathscr{A}$, we have wdb $\mathscr{A} \leq \mathrm{db} \mathscr{A}$ (see [7, VII, Section 3.4] and [13]).

A consequence of the main results of this paper (Theorem 2.2 and Theorem 3.4) is that the weak homological bi-dimension of $c_{0}\left(\mathbb{Z}, \omega^{-1}\right)$ is infinity, that is,

$$
w \mathrm{db} c_{0}\left(\mathbb{Z}, \omega^{-1}\right)=\infty
$$

The paper is organized as follows. In Section 2 we calculate the even dimensional cyclic and Hochschild cohomology groups of $\mathscr{A}^{\#}$ with coefficients in $\left(\mathscr{A}^{\#}\right)^{\prime}$, the dual space of $\mathscr{A}^{\#}$. In Section 3 we will continue our argument for the odd dimensional case. 


\section{Even dimensional cohomology groups of weighted sequence algebras}

In this section we prove that $\mathscr{H}^{2 n}\left(\mathscr{A}^{\#},\left(\mathscr{A}^{\#}\right)^{\prime}\right) \neq 0$ and $\mathscr{H}_{\mathscr{C}^{2 n}}\left(\mathscr{A}^{*}\right) \neq 0$ for every $n \in \mathbb{N}$.

LEMMA 2.1. Let $\sum_{i=-\infty}^{\infty} \alpha_{i}$ be an absolutely convergent series of real numbers, and let

$$
\phi: \overbrace{\mathscr{A}^{\#} \times \mathscr{A}^{\#} \times \cdots \times \mathscr{A}^{\#}}^{2 n \text { imes }} \rightarrow\left(\mathscr{A}^{\#}\right)^{\prime}
$$

be the function defined by

$$
\phi\left(a_{1}, \ldots, a_{2 n}\right)\left(a_{2 n+1}\right)=\sum_{i=-\infty}^{\infty} \frac{a_{1 i}^{\prime} \cdots a_{(2 n+1) i}^{\prime}}{\omega(i)^{(2 n+1)}} \alpha_{i}
$$

where $a_{k}=a_{k}^{\prime}+\beta_{k} 1$ and $a_{k}^{\prime}=\left\{a_{k i}^{\prime}\right\}_{i \in \mathbb{Z}}(k=1,2, \ldots, 2 n+1)$. Then $\phi$ is a bounded cyclic $2 n$-cocycle for every $n \in \mathbb{N}$.

PROOF. It is easy to see that $\phi$ is a $2 n$-linear map. Also

$$
\begin{aligned}
\left|\phi\left(a_{1}, \ldots, a_{2 n}\right)\left(a_{2 n+1}\right)\right| & \leq \sum_{i=-\infty}^{\infty} \frac{\left|a_{1 i}^{\prime} \cdots a_{(2 n+1) i}^{\prime}\right|}{\omega(i)^{2 n+1}}\left|\alpha_{i}\right| \\
& \leq \sup _{i}\left\{\frac{\left|a_{1 i}^{\prime}\right|}{\omega(i)}\right\} \cdots \sup _{i}\left\{\frac{\left|a_{(2 n+1) i}^{\prime}\right|}{\omega(i)}\right\}\left(\sum_{i=-\infty}^{\infty}\left|\alpha_{i}\right|\right) \\
& \leq\left\|a_{1}\right\|_{\omega^{-1}} \cdots\left\|a_{2 n+1}\right\|_{\omega^{-1}}\left(\sum_{i=-\infty}^{\infty}\left|\alpha_{i}\right|\right) .
\end{aligned}
$$

Thus $\phi$ is bounded and $\|\phi\| \leq \sum_{i=-\infty}^{\infty}\left|\alpha_{i}\right|$. Now we want to show that $\phi$ is a $2 n$-cocycle, that is,

$$
\begin{aligned}
\delta \phi\left(a_{1}, \ldots, a_{2 n+1}\right)\left(a_{2 n+2}\right)= & a_{1} \phi\left(a_{2}, \ldots, a_{2 n+1}\right)\left(a_{2 n+2}\right) \\
& +\sum_{i=1}^{2 n}(-1)^{i} \phi\left(a_{1}, \ldots, a_{i} a_{i+1}, \ldots, a_{2 n+1}\right)\left(a_{2 n+2}\right) \\
& +(-1)^{2 n+1}\left(\phi\left(a_{1}, \ldots, a_{2 n}\right) a_{2 n+1}\right)\left(a_{2 n+2}\right)=0 .
\end{aligned}
$$

Now we calculate all terms on the right-hand side of the above equation and we obtain the following $(2 n+2)$ terms respectively;

$$
\sum_{i=-\infty}^{\infty} \frac{\alpha_{i}}{\omega(i)^{2 n+1}}\left\{a_{1 i}^{\prime} \cdots a_{(2 n+2) i}^{\prime}+\beta_{1} a_{2 i}^{\prime} \cdots a_{(2 n+2) i}^{\prime}+\beta_{(2 n+2)} a_{1 i}^{\prime} \cdots a_{(2 n+1) i}^{\prime}\right.
$$




$$
\begin{aligned}
& -\left(a_{1 i}^{\prime} \cdots a_{(2 n+2) i}^{\prime}+\beta_{1} a_{2 i}^{\prime} \cdots a_{(2 n+2) i}^{\prime}+\beta_{2} a_{1 i}^{\prime} a_{3 i}^{\prime} \cdots a_{(2 n+2) i}^{\prime}\right) \\
& \pm \\
& +\left(a_{1 i}^{\prime} \cdots a_{(2 n+2) i}^{\prime}+\beta_{2 n} a_{1 i}^{\prime} \cdots a_{(2 n-1) i}^{\prime} a_{(2 n+1) i}^{\prime} a_{(2 n+2) i}^{\prime}\right. \\
& \left.+\beta_{(2 n+1)} a_{1 i}^{\prime} \cdots a_{(2 n) i}^{\prime} a_{(2 n+2) i}^{\prime}\right) \\
& \left.-\left(a_{1 i}^{\prime} \cdots a_{(2 n+2) i}^{\prime}+\beta_{(2 n+1)} a_{1 i}^{\prime} \cdots a_{(2 n) i}^{\prime} a_{(2 n+2) i}^{\prime}+\beta_{(2 n+2)} a_{1 i}^{\prime} \cdots a_{(2 n+1) i}^{\prime}\right)\right\} \text {. }
\end{aligned}
$$

So all terms in the above equation cancel in pairs. Thus $\phi$ is a $2 n$-cocycle, and obviously it is cyclic, that is,

$$
\phi\left(a_{1}, \ldots, a_{2 n}\right)\left(a_{2 n+1}\right)=(-1)^{2 n} \phi\left(a_{2}, \ldots, a_{2 n}, a_{2 n+1}\right)\left(a_{1}\right) .
$$

THEOREM 2.2. Let $\omega$ be a weight on $\mathbb{Z}$ such that $\inf \{\omega(i)\}=0$. Then

$$
\mathscr{H}^{2 n}\left(\mathscr{A}^{\#},\left(\mathscr{A}^{\#}\right)^{\prime}\right) \neq 0 \quad \text { and also } \quad \mathscr{H}^{2 n}\left(\mathscr{A}^{\#}\right) \neq 0
$$

for every $n \in \mathbb{N}$.

PROOF. Let $\phi$ be the bounded cyclic $2 n$-cocycle which was introduced in Lemma 2.1 and let $\alpha_{i}$ be defined as below. Since $\inf \{\omega(i)\}=0$, then there exist numbers $m_{k}$, $(k=1,2, \ldots)$ such that $m_{i} \neq m_{j}$ whenever $i \neq j$ and $\omega\left(m_{k}\right) \leq 1 / 2^{k}$. Now we define

$$
\alpha_{i}=\left\{\begin{array}{ll}
1 / k^{2} & \text { if } i=m_{k} \\
0 & \text { otherwise }
\end{array} \quad(k=1,2, \ldots) ;\right.
$$

and so $\sum_{i=-\infty}^{\infty} \alpha_{i}=\sum_{k=1}^{\infty} 1 / k^{2}$ which converges. Thus by Lemma 2.1

$$
\phi\left(a_{1}, \ldots, a_{2 n}\right)\left(a_{2 n+1}\right)=\sum_{k=1}^{\infty} \frac{a_{1 m_{k}}^{\prime} \cdots a_{(2 n+1) m_{k}}^{\prime}}{\omega\left(m_{k}\right)^{2 n+1} k^{2}}
$$

is a bounded cyclic $2 n$-cocycle for every $n \in \mathbb{N}$. Now if there exists a $\psi$ in $\mathscr{C}^{2 n-1}\left(\mathscr{A}^{\#},\left(\mathscr{A}^{\#}\right)^{\prime}\right)$ such that

$$
\begin{aligned}
\phi\left(a_{1}, \ldots, a_{2 n}\right)\left(a_{2 n+1}\right)= & \delta \psi\left(a_{1}, \ldots, a_{2 n}\right)\left(a_{2 n+1}\right) \\
= & a_{1} \psi\left(a_{2}, \ldots, a_{2 n}\right)\left(a_{2 n+1}\right) \\
& +\sum_{i=1}^{2 n-1}(-1)^{i} \psi\left(a_{1}, \ldots, a_{i} a_{i+1}, \ldots, a_{2 n}\right)\left(a_{2 n+1}\right) \\
& +(-1)^{2 n}\left(\psi\left(a_{1}, \ldots, a_{2 n-1}\right) a_{2 n}\right)\left(a_{2 n+1}\right),
\end{aligned}
$$


where $a_{k} \in \mathscr{A}^{*}(k=1,2, \ldots, 2 n+1)$, in particular, if $a_{1}=\cdots=a_{2 n+1}=e_{m_{j}}$, $(j=1,2, \ldots)$, then

$$
\phi(\overbrace{e_{m_{j}}, \ldots, e_{m_{j}}}^{2 n \text { tumes }})\left(e_{m_{j}}\right)=\psi(\overbrace{e_{m_{j}}, \ldots, e_{m_{j}}}^{2 n-1 \text { times }})\left(e_{m_{j}}\right)=\frac{1}{\omega\left(m_{j}\right)^{2 n+1} j^{2}} .
$$

So since $\omega(j) \leq 1 / 2^{j}$

$$
\begin{aligned}
\|\psi\| & \geq \sup _{j}\left\{\mid \psi(\overbrace{\omega\left(m_{j}\right) e_{m_{j}}, \ldots, \omega\left(m_{j}\right) e_{m_{j}}}^{2 n \text { times }}\left(\omega\left(m_{j}\right) e_{m_{j}}\right) \mid\}\right. \\
& =\sup _{j}\left\{\frac{\omega\left(m_{j}\right)^{2 n}}{\omega\left(m_{j}\right)^{2 n+1} j^{2}}\right\}=\sup _{j}\left\{\frac{1}{\omega\left(m_{j}\right) j^{2}}\right\} \geq \sup _{j}\left\{\frac{2^{j}}{j^{2}}\right\}=\infty
\end{aligned}
$$

which is a contradiction. So $\mathscr{H}^{2 n}\left(\mathscr{A}^{\#},\left(\mathscr{A}^{\#}\right)^{\prime}\right) \neq 0$ and also $\mathscr{H}^{2 n}\left(\mathscr{A}^{\#}\right) \neq 0$.

\section{Odd dimensional cohomology groups of weighted sequence algebras}

In this section we will show that $\mathscr{H}^{2 n+1}\left(\mathscr{A}^{\#},\left(\mathscr{A}^{\#}\right)^{\prime}\right) \neq 0$ and also $\mathscr{H} \mathscr{C}^{2 n+1}\left(\mathscr{A}^{\#}\right) \neq 0$ for every $n \geq 1$. Note that the structure of the function $\phi$ which is a base for Theorem 3.4, for the three dimensional case is different from the structure of the corresponding functions in the other cases.

LEMMA 3.1. Let $\sum_{i=-\infty}^{\infty} \alpha_{i}$ be an absolutely convergent series of real numbers, and let $\phi: \mathscr{A}^{\#} \times \mathscr{A}^{\#} \times \mathscr{A}^{\#} \rightarrow\left(\mathscr{A}^{*}\right)^{\prime}$ be the function defined by

$$
\phi(a, b, c)(d)=\sum_{i=-\infty}^{\infty} \sum_{j=-\infty}^{\infty} \frac{a_{j}^{\prime} b_{i}^{\prime} c_{i}^{\prime} d_{j}^{\prime}-a_{i}^{\prime} b_{i}^{\prime} c_{j}^{\prime} d_{j}^{\prime}}{\omega(i)^{2} \omega(j)^{2}} \alpha_{i} \alpha_{j}
$$

where $a=a^{\prime}+\alpha 1, b=b^{\prime}+\beta 1, c=c^{\prime}+\gamma 1$ and $d=d^{\prime}+\lambda 1$. Then $\phi$ is a bounded cyclic 3-cocycle.

PROOF. It is easy to see that $\phi$ is a trilinear map and also

$$
\begin{aligned}
|\phi(a, b, c)(d)| & \leq \sum_{i=-\infty}^{\infty} \sum_{j=-\infty}^{\infty} \frac{\left|a_{j}^{\prime} b_{i}^{\prime} c_{i}^{\prime} d_{j}^{\prime}\right|+\left|a_{i}^{\prime} b_{i}^{\prime} c_{j}^{\prime} d_{j}^{\prime}\right|}{\omega(i)^{2} \omega(j)^{2}}\left|\alpha_{i}\right|\left|\alpha_{j}\right| \\
& \leq 2\|a\|_{\omega^{-1}}\|b\|_{\omega^{-1}}\|c\|_{\omega^{-1}}\|d\|_{\omega^{-1}}\left(\sum_{i=-\infty}^{\infty}\left|\alpha_{i}\right|\right)^{2}
\end{aligned}
$$


Thus $\phi$ is bounded and $\|\phi\| \leq 2\left\{\sum_{i=-\infty}^{\infty}\left|\alpha_{i}\right|\right\}^{2}$. Now we want to show that $\phi$ satisfies

$$
\begin{aligned}
a \phi(b, c, d)(h) & -\phi(a b, c, d)(h)+\phi(a, b c, d)(h) \\
& -\phi(a, b, c d)(h)+(\phi(a, b, c) d)(h)=0,
\end{aligned}
$$

where $a=a^{\prime}+\alpha 1, b=b^{\prime}+\beta 1, c=c^{\prime}+\gamma 1, d=d^{\prime}+\lambda 1$ and $h=h^{\prime}+\theta 1$. By definition of $\phi$ and (1)

$$
\begin{aligned}
& \sum_{i} \sum_{j} \frac{\alpha_{i} \alpha_{j}}{\omega(i)^{2} \omega(j)^{2}}(\left\{\left(b_{j}^{\prime} c_{i}^{\prime} d_{i}^{\prime} h_{j}^{\prime} a_{j}^{\prime}+\alpha b_{j}^{\prime} c_{i}^{\prime} d_{i}^{\prime} h_{j}^{\prime}+\theta b_{j}^{\prime} c_{i}^{\prime} d_{i}^{\prime} a_{j}^{\prime}\right)\right. \\
&\left.-\left(b_{i}^{\prime} c_{i}^{\prime} d_{j}^{\prime} h_{j}^{\prime} a_{j}^{\prime}+\alpha b_{i}^{\prime} c_{i}^{\prime} d_{j}^{\prime} h_{j}^{\prime}+\theta b_{i}^{\prime} c_{i}^{\prime} d_{j}^{\prime} a_{j}^{\prime}\right)\right\} \\
&-\left\{\left(a_{j}^{\prime} b_{j}^{\prime} c_{i}^{\prime} d_{i}^{\prime} h_{j}^{\prime}+\alpha b_{j}^{\prime} c_{i}^{\prime} d_{i}^{\prime} h_{j}^{\prime}+\beta a_{j}^{\prime} c_{i}^{\prime} d_{i}^{\prime} h_{j}^{\prime}\right)\right. \\
&\left.-\left(a_{i}^{\prime} b_{i}^{\prime} c_{i}^{\prime} d_{j}^{\prime} h_{j}^{\prime}+\alpha b_{i}^{\prime} c_{i}^{\prime} d_{j}^{\prime} h_{j}^{\prime}+\beta a_{i}^{\prime} c_{i}^{\prime} d_{j}^{\prime} h_{j}^{\prime}\right)\right\} \\
&+\left\{\left(a_{j}^{\prime} b_{i}^{\prime} c_{i}^{\prime} d_{i}^{\prime} h_{j}^{\prime}+\beta a_{j}^{\prime} c_{i}^{\prime} d_{i}^{\prime} h_{j}^{\prime}+\gamma a_{j}^{\prime} b_{i}^{\prime} d_{i}^{\prime} h_{j}^{\prime}\right)\right. \\
&\left.-\left(a_{i}^{\prime} b_{i}^{\prime} c_{i}^{\prime} d_{j}^{\prime} h_{j}^{\prime}+\beta a_{i}^{\prime} c_{i}^{\prime} d_{j}^{\prime} h_{j}^{\prime}+\gamma a_{i}^{\prime} b_{i}^{\prime} d_{j}^{\prime} h_{j}^{\prime}\right)\right\} \\
&-\left\{\left(a_{j}^{\prime} b_{i}^{\prime} c_{i}^{\prime} d_{i}^{\prime} h_{j}^{\prime}+\gamma a_{j}^{\prime} b_{i}^{\prime} d_{i}^{\prime} h_{j}^{\prime}+\lambda a_{j}^{\prime} b_{i}^{\prime} c_{i}^{\prime} h_{j}^{\prime}\right)\right. \\
&\left.-\left(a_{i}^{\prime} b_{i}^{\prime} c_{j}^{\prime} d_{j}^{\prime} h_{j}^{\prime}+\gamma a_{i}^{\prime} b_{i}^{\prime} d_{j}^{\prime} h_{j}^{\prime}+\lambda a_{i}^{\prime} b_{i}^{\prime} c_{j}^{\prime} h_{j}^{\prime}\right)\right\} \\
&+\left\{\left(a_{j}^{\prime} b_{i}^{\prime} c_{i}^{\prime} d_{j}^{\prime} h_{j}^{\prime}+\lambda a_{j}^{\prime} b_{i}^{\prime} c_{i}^{\prime} h_{j}^{\prime}+\theta a_{j}^{\prime} b_{i}^{\prime} c_{i}^{\prime} d_{j}^{\prime}\right)\right. \\
&\left.\left.-\left(a_{i}^{\prime} b_{i}^{\prime} c_{j}^{\prime} d_{j}^{\prime} h_{j}^{\prime}+\lambda a_{i}^{\prime} b_{i}^{\prime} c_{j}^{\prime} h_{j}^{\prime}+\theta a_{i}^{\prime} b_{i}^{\prime} c_{j}^{\prime} d_{j}^{\prime}\right)\right\}\right) \\
&=\sum_{i} \sum_{j} \frac{\theta b_{j}^{\prime} c_{i}^{\prime} d_{i}^{\prime} a_{j}^{\prime}}{\omega(i)^{2} \omega(j)^{2}} \alpha_{i} \alpha_{j}-\sum_{i} \sum_{j} \frac{\theta a_{i}^{\prime} b_{i}^{\prime} c_{j}^{\prime} d_{j}^{\prime}}{\omega(i)^{2} \omega(j)^{2}} \alpha_{i} \alpha_{j}=0
\end{aligned}
$$

and so $\phi$ is a 3 -cocycle. Also $\phi$ is cyclic, since

$$
\begin{aligned}
\phi(d, a, b, c) & =\sum_{i} \sum_{j} \frac{d_{j}^{\prime} a_{i}^{\prime} b_{i}^{\prime} c_{j}^{\prime}-d_{i}^{\prime} a_{i}^{\prime} b_{j}^{\prime} c_{j}^{\prime}}{\omega(i)^{2} \omega(j)^{2}} \alpha_{i} \alpha_{j} \\
& =-\sum_{i} \sum_{j} \frac{a_{i}^{\prime} b_{j}^{\prime} c_{j}^{\prime} d_{i}^{\prime}}{\omega(i)^{2} \omega(j)^{2}} \alpha_{i} \alpha_{j}+\sum_{i} \sum_{j} \frac{a_{i}^{\prime} b_{i}^{\prime} c_{j}^{\prime} d_{j}^{\prime}}{\omega(i)^{2} \omega(j)^{2}} \alpha_{i} \alpha_{j} \\
& =-\phi(a, b, c, d)=(-1)^{3} \phi(a, b, c, d) .
\end{aligned}
$$

Now we are going to construct the $2 n+1$-cocycle $\phi$ for higher dimensions.

$$
2 n \text { times }
$$

LEMMA 3.2. Let $\psi_{i j}: \overbrace{\mathscr{A}^{\#} \times \mathscr{A}^{\#} \times \cdots \times \mathscr{A}^{\#}} \rightarrow\left(\mathscr{A}^{\#}\right)^{\prime}$ be a 2 -linear function defined by

$$
\psi_{i j}\left(a_{1}, \ldots, a_{2 n}\right)\left(a_{2 n+1}\right)=\sum_{k=1}^{2 n+1} a_{1 i}^{\prime} \cdots a_{k j}^{\prime} \cdots a_{(2 n+1) i}^{\prime}
$$


where $a_{k}=a_{k}^{\prime}+\beta_{k} 1$ and $a_{k}^{\prime}=\left\{a_{k i}^{\prime}\right\}_{i \in \mathbb{Z}}(k=1, \ldots, 2 n+1)$. Then

$$
\begin{aligned}
\delta \psi_{i j}\left(a_{1}, \ldots, a_{2 n+1}\right)\left(a_{2 n+2}\right)= & a_{1 j}^{\prime} a_{2 i}^{\prime} \cdots a_{(2 n+1) i}^{\prime} a_{(2 n+2) j}^{\prime} \\
& +\sum_{k=1}^{2 n+1}(-1)^{k} a_{1 i}^{\prime} \cdots a_{k j}^{\prime} a_{(k+1) j}^{\prime} \cdots a_{(2 n+2) i}^{\prime} .
\end{aligned}
$$

PROOF. By the coboundary formula we have

(2) $\delta \psi_{i j}\left(a_{1}, \ldots, a_{2 n+1}\right)\left(a_{2 n+2}\right)=\psi_{i j}\left(a_{2}, \ldots, a_{2 n+1}\right)\left(a_{2 n+2} a_{1}\right)$

$$
\begin{aligned}
& +\sum_{k=1}^{2 n}(-1)^{k} \psi_{i j}\left(a_{1}, \ldots, a_{k} a_{k+1}, \ldots, a_{2 n+1}\right)\left(a_{2 n+2}\right) \\
& -\psi_{i j}\left(a_{1}, \ldots, a_{2 n}\right)\left(a_{2 n+1} a_{2 n+2}\right) .
\end{aligned}
$$

Using the definition of $\psi_{i j}$ we obtain the value of all terms on the right-hand side of the above equation as follows

$$
\begin{aligned}
& \psi_{i j}\left(a_{2}, \ldots, a_{2 n+1}\right)\left(a_{2 n+2} a_{1}\right) \\
&=a_{2 i}^{\prime} \cdots a_{(2 n+1) i}^{\prime} a_{(2 n+2) j}^{\prime} a_{1 j}^{\prime}+\sum_{k=2}^{2 n+1} a_{1 i}^{\prime} \cdots a_{k j}^{\prime} \cdots a_{(2 n+2) i}^{\prime} \\
&+\sum_{k=2}^{2 n+2} \beta_{1} a_{2 i}^{\prime} \cdots a_{k j}^{\prime} \cdots a_{(2 n+2) i}^{\prime}+\sum_{k=1}^{2 n+1} \beta_{2 n+2} a_{1 i}^{\prime} \cdots a_{k j}^{\prime} \cdots a_{(2 n+1) i}^{\prime} .
\end{aligned}
$$

For $l=1, \ldots, 2 n$,

$$
\begin{aligned}
\psi_{i j} & \left(a_{1}, \ldots, a_{l} a_{l+1}, \ldots, a_{2 n+1}\right)\left(a_{2 n+2}\right) \\
= & a_{1 i}^{\prime} \cdots a_{l j}^{\prime} a_{(l+1) j}^{\prime} \cdots a_{(2 n+2) i}^{\prime}+\sum_{\substack{k=1 \\
k \neq l, l+1}}^{2 n+2} a_{1 i}^{\prime} \cdots a_{k j}^{\prime} \cdots a_{(2 n+2) i}^{\prime} \\
& +\sum_{\substack{k=1 \\
k \neq l}}^{2 n+2} \beta_{l} a_{2 i}^{\prime} \cdots a_{k j}^{\prime} \cdots \widehat{a}_{l}^{\prime} \cdots a_{(2 n+2) i}^{\prime}+\sum_{\substack{k=1 \\
k \neq l+1}}^{2 n+2} \beta_{l+1} a_{1 i}^{\prime} \cdots a_{k j}^{\prime} \cdots \widehat{a}_{l+1}^{\prime} \cdots a_{(2 n+2) i}^{\prime},
\end{aligned}
$$

where symbol $\hat{?}$ shows the element in that position is removed.

$$
\begin{aligned}
& \psi_{i j}\left(a_{1}, \ldots, a_{2 n}\right)\left(a_{2 n+1} a_{2 n+2}\right) \\
& \quad=a_{1 i}^{\prime} \cdots a_{(2 n) i}^{\prime} a_{(2 n+1) j}^{\prime} a_{(2 n+2) j}^{\prime}+\sum_{k=1}^{2 n} a_{1 i}^{\prime} \cdots a_{k j}^{\prime} \cdots a_{(2 n+2) i}^{\prime}
\end{aligned}
$$




$$
+\sum_{\substack{k=1 \\ k \neq 2 n+1}}^{2 n+2} \beta_{2 n+1} a_{1 i}^{\prime} \cdots a_{k j}^{\prime} \cdots a_{(2 n) i}^{\prime} a_{(2 n+2) i}^{\prime}+\sum_{k=1}^{2 n+1} \beta_{2 n+2} a_{1 i}^{\prime} \cdots a_{k j}^{\prime} \cdots a_{(2 n+1) i}^{\prime}
$$

Substitute the values for $\psi_{i j}$ obtained above in (2). Then all summations with $\beta_{k}$ $(k=i, \ldots, 2 n+2)$ coefficients cancel in pairs, and we obtain

$$
\begin{aligned}
\delta \psi_{i j}\left(a_{1}, \ldots, a_{2 n+1}\right)\left(a_{2 n+2}\right) & \\
= & a_{1 j}^{\prime} a_{2 i}^{\prime} \cdots a_{(2 n+1) i}^{\prime} a_{(2 n+2) j}^{\prime}+\sum_{k=1}^{2 n+1}(-1)^{k} a_{1 i}^{\prime} \cdots a_{k j}^{\prime} a_{(k+1) j}^{\prime} \cdots a_{(2 n+2) i}^{\prime} \\
& +\sum_{k=2}^{2 n+1} a_{1 i}^{\prime} \cdots a_{k j}^{\prime} \cdots a_{(2 n+2) i}^{\prime}+\sum_{l=1}^{2 n+1}(-1)^{l} \sum_{\substack{k=1 \\
k \neq l, l+1}}^{2 n+2} a_{1 i}^{\prime} \cdots a_{k j}^{\prime} \cdots a_{(2 n+2) i}^{\prime}
\end{aligned}
$$

and the sum of the last two terms is zero because, they contain $2 n$ terms like $a_{1 i}^{\prime} \cdots a_{k j}^{\prime} \cdots a_{(2 n+2) i}^{\prime}$ for every $k=1, \ldots, 2 n+2$, half with a positive sign and the other half with a negative sign which cancel in pairs. So this finishes the proof.

LEMMA 3.3. Let $\sum_{i} \alpha_{i}$ be an absolutely convergent series of real numbers, and let $\phi: \underbrace{\mathscr{A}^{\#} \times \mathscr{A}^{\#} \times \cdots \times \mathscr{A}^{\#}}_{2 n+1 \text { times }} \rightarrow\left(\mathscr{A}^{\#}\right)^{\prime}$ be the function defined by

$$
\phi\left(a_{1}, \ldots, a_{2 n+1}^{2 n+1 \text { times }}\right)\left(a_{2 n+2}\right)=\sum_{i} \sum_{j} \frac{\alpha_{i} \alpha_{j}}{\omega(i)^{2 n} \omega(j)^{2}} \delta \psi_{i j}\left(a_{1}, \ldots, a_{2 n+1}\right)\left(a_{2 n+2}\right),
$$

where $\psi_{i j}$ is defined as in Lemma 3.2. Then $\phi$ is a bounded cyclic $(2 n+1)$-cocycle for every $n>1$.

Proof. It is easy to see that $\phi$ is a $2 n+1$-linear map and also

$$
\left|\phi\left(a_{1}, \ldots, a_{2 n+1}\right)\left(a_{2 n+2}\right)\right| \leq(2 n+2)\left\|a_{1}\right\|_{\omega^{-1}} \cdots\left\|a_{2 n+2}\right\|_{\omega^{-1}}\left(\sum_{i}\left|\alpha_{i}\right|\right)^{2}
$$

Thus $\phi$ is bounded and $\|\phi\| \leq(2 n+2)\left(\sum_{i=-\infty}^{\infty}\left|\alpha_{i}\right|\right)^{2}$. Also $\phi$ is a $(2 n+1)$-cocycle, that is,

$$
\delta \phi=\sum_{i} \sum_{j} \frac{\alpha_{i} \alpha_{j}}{\omega(i)^{2 n} \omega(j)^{2}} \delta \delta \psi_{i j}=0
$$

because $\delta \delta \psi_{i j}=0$. Furthermore we show that $\phi$ is cyclic, that is, it satisfies

$$
\phi\left(a_{1}, \ldots, a_{2 n+1}\right)\left(a_{2 n+2}\right)=(-1)^{2 n+1} \phi\left(a_{2}, \ldots, a_{2 n+2}\right)\left(a_{1}\right) .
$$


For this we have to calculate the right-hand side of the above equation. We have the following:

$$
\begin{aligned}
& \phi\left(a_{2}, \ldots, a_{2 n+2}\right)\left(a_{1}\right) \\
&=\sum_{i} \sum_{j}\left\{\frac { \alpha _ { i } \alpha _ { j } } { \omega ( i ) ^ { 2 n } \omega ( j ) ^ { 2 } } \left(a_{2 j}^{\prime} a_{3 i}^{\prime} \cdots a_{(2 n+2) i}^{\prime} a_{1 j}^{\prime}-a_{2 j}^{\prime} a_{3 j}^{\prime} \cdots a_{(2 n+2) i}^{\prime} a_{1 i}^{\prime}\right.\right. \\
&\left.\left.+a_{2 i}^{\prime} a_{3 j}^{\prime} a_{4 j}^{\prime} \cdots a_{(2 n+2) i}^{\prime} a_{1 i}^{\prime} \mp \cdots-a_{2 i}^{\prime} \cdots a_{(2 n+1) i}^{\prime} a_{(2 n+2) j}^{\prime} a_{1 j}^{\prime}\right)\right\} \\
&= \sum_{i} \sum_{j}\left\{\frac { \alpha _ { i } \alpha _ { j } } { \omega ( i ) ^ { 2 n } \omega ( j ) ^ { 2 } } \left(-a_{1 j}^{\prime} a_{2 i}^{\prime} \cdots a_{(2 n+1) i}^{\prime} a_{(2 n+2) j}^{\prime}+a_{1 j}^{\prime} a_{2 j}^{\prime} a_{3 i}^{\prime} \cdots a_{(2 n+2) i}^{\prime}\right.\right. \\
&\left.\left.\mp \cdots-a_{1 i}^{\prime} \cdots a_{(2 n) j}^{\prime} a_{(2 n+1) j}^{\prime} a_{(2 n+2) i}^{\prime}+a_{1 i}^{\prime} \cdots a_{(2 n) i}^{\prime} a_{(2 n+1) j}^{\prime} a_{(2 n+2) j}^{\prime}\right)\right\} \\
&=-\phi\left(a_{1}, \ldots, a_{2 n+1}\right)\left(a_{2 n+2}\right) .
\end{aligned}
$$

Therefore $\phi$ is a cyclic $(2 n+1)$-cocycle.

THEOREM 3.4. Let $\omega$ be a weight on $\mathbb{Z}$ such that $\inf \{\omega(i)\}=0$. Then

$$
\mathscr{H}^{2 n+1}\left(\mathscr{A}^{\#},\left(\mathscr{A}^{\#}\right)^{\prime}\right) \neq 0
$$

and also $\mathscr{H} \mathscr{C}^{2 n+1}\left(\mathscr{A}^{\#}\right) \neq 0$ for every $n \in \mathbb{N}$.

PROOF. Let $\phi$ be the bounded $2 n+1$-cocycle which was introduced in Lemma 3.1 for $n=1$ and in Lemma 3.3 for $n>1$. Consider the sequence $\alpha_{i}$ which was defined in the proof of Theorem 2.2. Note that $m_{i} \neq m_{j}$ whenever $i \neq j$ and $\omega\left(m_{k}\right) \leq 1 / 2^{k}$. Also if $i<j$, since $1 / 2^{j}<1 / 2^{i}$, then $\max \left\{\omega\left(m_{i}\right), \omega\left(m_{j}\right)\right\} \leq 1 / 2^{i}$.

Now if $\psi \in \mathscr{C}^{2 n}\left(\mathscr{A}^{\#},\left(\mathscr{A}^{\#}\right)^{\prime}\right)$ such that $\phi=\delta \psi$, then by the definition of $\phi$ and the coboundary formula we have

$$
\begin{aligned}
\phi\left(e_{m_{j}},\right. & \overbrace{e_{m_{i}}, \ldots, e_{m_{i}}}^{2 n \text { times }})\left(e_{m_{j}}\right)=\psi(\overbrace{e_{m_{i}}, \ldots, e_{m_{i}}}^{2 n \text { times }})\left(e_{m_{j}}\right) \\
& +\overbrace{\psi(e_{m_{j}}, \underbrace{e_{m_{i}}, \ldots, e_{m_{i}}}_{2 n-1 \text { times }})\left(e_{m_{j}}\right) \pm \ldots+\psi(e_{m_{j}}, \underbrace{e_{m_{i}}, \ldots, e_{m_{i}}}_{2 n-1 \text { times }})\left(e_{m_{j}}\right)}^{2 n-1 \text { times }} \\
& =\psi\left(e_{m_{i}}, \ldots, e_{m_{i}}\right)\left(e_{m_{j}}\right)+\psi\left(e_{m_{j}}, e_{m_{i}}, \ldots, e_{m_{i}}\right)\left(e_{m_{j}}\right) \\
& =\psi(e_{m_{i}}+e_{m_{j}}, \overbrace{e_{m_{i}}, \ldots, e_{m_{i}}}^{2 n-1 \text { times }})\left(e_{m_{j}}\right) .
\end{aligned}
$$

Therefore by the definition of $\phi$

$$
\psi(e_{m_{i}}+e_{m_{j}}, \overbrace{e_{m_{i}}, \ldots, e_{m_{i}}}^{2 n-1 \text { times }})\left(e_{m_{j}}\right)=\frac{\alpha_{m_{i}} \alpha_{m_{j}}}{\omega\left(m_{i}\right)^{2 n} \omega\left(m_{j}\right)^{2}} .
$$


Suppose $\min \left\{\omega\left(m_{i}\right), \omega\left(m_{j}\right)\right\}=C_{i j}$, then

$$
\left\|C_{i j}\left(e_{m_{\mathrm{i}}}+e_{m_{j}}\right)\right\|_{\omega^{-1}}=1 \text { and }\left\|\omega\left(m_{i}\right) e_{m_{i}}\right\|_{\omega^{-1}}=1 .
$$

If we let $i<j$, then

$$
\begin{aligned}
\|\psi\| & \geq \sup _{i, j}\left\{\left|\psi\left(C_{i j}\left(e_{m_{i}}+e_{m_{j}}\right), \omega\left(m_{i}\right) e_{m_{i}}, \ldots, \omega\left(m_{i}\right) e_{m_{i}}\right)\left(\omega\left(m_{j}\right) e_{m_{j}}\right)\right|\right\} \\
& =\sup _{i, j}\left\{\frac{\min \left\{\omega\left(m_{i}\right), \omega\left(m_{j}\right)\right\} \alpha_{m_{i}} \alpha_{m_{j}}}{\omega\left(m_{i}\right) \omega\left(m_{j}\right)}\right\} \\
& =\sup _{i, j}\left\{\frac{1}{\max \left\{\omega\left(m_{i}\right), \omega\left(m_{j}\right)\right\} i^{2} j^{2}}\right\} \geq \sup _{i, j}\left\{\frac{2^{i}}{j^{4}}\right\} .
\end{aligned}
$$

In particular, for $j=i+1$, we have $\|\psi\| \geq \sup _{i} 2^{i} /(i+1)^{4}=\infty$ which contradicts $\psi \in \mathscr{C}^{2 n}\left(\mathscr{A}^{\#},\left(\mathscr{A}^{\#}\right)^{\prime}\right)$. So $\mathscr{H}^{2 n+1}\left(\mathscr{A}^{\#},\left(\mathscr{A}^{\#}\right)^{\prime}\right) \neq 0$ and $\mathscr{H} \mathscr{C}^{2 n+1}\left(\mathscr{A}^{\#}\right) \neq 0$.

REMARK. Consider the short exact sequence $0 \rightarrow \mathscr{A} \rightarrow \mathscr{A}^{\#} \rightarrow \mathbb{C} \rightarrow 0$. The dual of this short exact sequence, is the short exact sequence,

$$
0 \rightarrow \mathbb{C} \rightarrow\left(\mathscr{A}^{\#}\right)^{\prime} \rightarrow \mathscr{A}^{\prime} \rightarrow 0 .
$$

This gives the long exact sequence of cohomology (see [6, III. Corollary 4.11])

$$
\cdots \rightarrow \mathscr{H}^{n}\left(\mathscr{A}^{\#}, \mathbb{C}\right) \rightarrow \mathscr{H}^{n}\left(\mathscr{A}^{\#},\left(\mathscr{A}^{\#}\right)^{\prime}\right) \rightarrow \mathscr{H}^{n}\left(\mathscr{A}^{\#}, \mathscr{A}^{\prime}\right) \rightarrow \cdots
$$

From this, one can show that $\mathscr{H}^{n}\left(\mathscr{A}^{\#}, \mathbb{C}\right) \neq 0$ for every $n \geq 2$.

As we noticed in Section $1, E_{N}$ is an amenable closed subalgebra of $\mathscr{A}^{\#}$. So $\mathscr{A}^{\#}$ satisfies the conditions of [12, Theorem 2.6 and Theorem 5.1]. We can therefore apply Theorem 2.2 and Theorem 3.4 to conclude that for each $n \geq 2$, the $E_{N}$-relative (cyclic) cohomology of $\mathscr{A}^{\#}$ does not vanish.

\section{Acknowledgment}

The author wishes to thank the referee for bringing the last Remark to his attention.

\section{References}

[1] W. G. Bade, P. C. Curtis, Jr. and H. G. Dales, 'Amenability and weak amenability for Beurling and Lipschitz algebras', Proc. London Math. Soc. 55 (1987), 359-377. 
[2] H. G. Dales and J. Duncan, 'Second order cohomology groups of some semigroup algebras', in: Banach Algebra'97 (Blaubeuren) (Walter de Gruyter, Berlin, 1998) pp. 101-117.

[3] H. G. Dales, F. Ghahramani and N. Grønbæk, 'Derivation into iterated duals of Banach algebras', Studia Math. 128 (1998), 19-54.

[4] F. Gourdeau and M. C. White, 'Vanishing of the third simplicial cohomology group of $\ell^{1}\left(Z_{+}\right)$', Trans. Amer. Math. Soc. 353 (2001), 2003-2017.

[5] N. Grønbæk, 'Weak and cyclic amenability for non-commutative Banach algebras', Proc. Edinburgh Math. Soc. 35 (1992), 315-328.

[6] A. Ya. Helemskii, The homology of Banach and topological algebras (Kluwer Academic Publishers, Dordrecht, 1986).

[7] - Banach and locally convex algebras (Oxford University Press, Oxford, 1993).

[8] B. E. Johnson, Cohomology in Banach algebras, Mem. Amer. Math. Soc. 127 (Amer. Math. Soc. Providence, 1972).

[9] — Derivations from $L^{1}(G)$ into $L^{1}(G)$ and $L^{\infty}(G)$, Lecture Notes in Math. 1359 (Springer, Berlin, 1988) pp. 191-198.

[10] - 'Alternating cohomology', preprint.

[11] B. E. Johnsona, R. V. Kadison and J. R. Ringrose, 'Cohomology of operator algebra III. Reduction to normal cohomology', Bull. Soc. Math. France 100 (1972), 73-96.

[12] Z. A. Lykova, 'Relative cohomology of Banach algebras', J. Operator Theory 41 (1999), 23-53.

[13] Yu. V. Selivanov, 'Weak homological bi-dimension and its values in the class of biflat Banach algebras', Extracta Math. 11 (1996), 348-365.

[14] A. M. Sinclair and R. R. Smith, Hochschild cohomology of von Neumann algebras, London Math. Soc. Lecture Note Ser. 204 (Cambridge Univ. Press, Cambridge, 1995) pp. 196.

Faculty of Mathematics and Computer Science

Amir Kabir University

$424 \mathrm{Hafez}$ Avenue

Tehran 15914

Iran

e-mail: arpabbas@aut.ac.ir 